spinal lesions on MRI are not as predictive of NMO in children as in adults. (Banwell B, Tenembaum S, Lennon VA et al. Neuromyelitis optica-IgG in childhood inflammatory demyelinating CNS disorders. Neurology January 29, 2008;70:344-352). (Reprints: Dr Sean Pittock, Department of Neurology, Mayo Clinic, Rochester, MN 55905).

COMMENT. Neuromyelitis optica is characterized by monophasic or recurrent episodes of optic neuritis and longitudinally extensive transverse myelitis, either monophasic or recurrent. The autoantibody NMO-IgG is present in $73 \%$ of adults with $\mathrm{NMO}$ and is $92 \%$ specific for NMO and related disorders, recurrent optic neuritis or transverse myelitis. In children with NMO in the above study, $47 \%$ were seropositive. The role of NMO-IgG autoantibody in NMO is unknown, but it may be important in recurrent disease. An overview of NMO in children is provided in an editorial (Levy M et al. Neurology Jan 2008;70:334$335)$.

The most useful diagnostic feature of NMO or NMO-spectrum disorders in adults is a longitudinally extensive spinal cord lesion. This contrasts with the well-circumscribed foci of increased T2-weighted signal in multiple sclerosis, typically seen in adults. In children with MS, the discrete spinal lesions are common, but $14 \%$ also show the longitudinally extensive spinal lesions, rare in adults with MS. A longitudinally extensive spinal lesion in a child with demyelinating disease does not exclude a diagnosis of MS and is less predictive of an NMOspectrum disorder than in adult patients.

Relationship between NMO and autoimmune disease. The association of NMO-IgG and non-organ-specific autoantibodies in patients with systemic lupus erythematosis (SLE) and in those with NMO spectrum disorder was evaluated at the Mayo Clinic and University of Lille, France (Pittock SJ et al. Arch Neurol 65:78-83). Patients with NMO were seropositive for NMO-IgG, and those with SLE without NMO were seronegative for this autoantibody. NMO-IgG is specific for distinguishing NMO spectrum disorder from multisystem autoimmune disorders. NMO may coexist with SLE and other autoimmune disease and is not a complication of SLE.

\title{
CORTICAL DEMYELINATION IN MULTIPLE SCLEROSIS
}

Cortical demyelination in CNS inflammatory demyelinating diseases is reviewed in an editorial and in a study by Moll NM et al (Neurology 2008;70:336-343). Cortical demyelination occurs in 3 different patterns: 1) leukocortical lesions, affecting both gray and white matter; 2) small perivenous intracortical lesions; and 3) widespread subpial demyelination. Type 3 is most abundant in multiple sclerosis (MS) and is related to chronic inflammation of the meninges. Cortical lesions in progressive multifocal leukoencephalopathy (PML) are similar to those in MS but are absent in HIV-encephalitis and adrenoleukodystrophy. T-cell inflammation is sparse in MS and PML cortical lesions in contrast to white matter lesions. Widespread subpial demyelination may be pathognomonic for MS and related inflammatory demyelinating diseases. (Lassmann $\mathrm{H}$, Lucchinetti CF. Cortical demyelination in CNS inflammatory demyelinating diseases. Neurology Jan 29, 2008;70:332-336). (Reprints: Prof Dr Hans Lassmann, Div Neuroimmunology, Centre for Brain Research, Medical University of Vienna, Spitalgasse 4, A-1090 Wien, Austria). 
COMMENT. Subpial cortical lesions in MS are related to an inflammatory process in the meninges. Cortical demyelination occurs mostly in progressive MS, and is invisible by conventional MRI.

Abnormal T-cell reactivities in childhood inflammatory demyelinating disease. (Banwell B et al. Ann Neurol Jan 2008;63:98-111). Peripheral T-cell proliferative responses to self-, dietary, and control antigens were evaluated in children with CNS inflammatory demyelination, recent-onset type 1 diabetes mellitus, nonautoimmune neurologic disorders, and healthy children. Those with inflammatory demyelination, CNS injury, and diabetes showed heightened T-cell reactivities to self-antigens. Nonspecific T-cell dysregulation is an early feature of childhood onset MS and diabetes. The study highlights the possible relation between dietary antigens (eg cow-milk reactivities) and autoimmune diseases. High milk consumption and early weaning to foreign protein diets have been proposed as a possible MS risk factor (Malosse D et al. Neuroepidemiology 1992;11:304-312). In the present study, the incidence of MS and diabetes was not different in patients exposed to infaint formula and those breast-fed exclusively, but the analysis was limited.

\section{DEGENERATIVE DISEASE}

\section{NEONATAL DIAGNOSIS AND TREATMENT OF MENKES DISEASE}

Infants diagnosed with Menkes disease early by plasma neurochemical methods and treated early, within 22 days after birth, with copper replacement therapy, had a $92 \%$ survival rate vs $13 \%$ in those treated late, Median follow-up in 12 newborns treated early was 4.6 years compared to 1.8 years in 15 diagnosed and treated late. Abnormally low copper dependent, dopamine-B-hydroxylase activity was identified by measuring plasma catecholamine levels in infants at risk. Response to treatment occurred only in patients with ATP7A mutations that permit some residual copper transport. (Kaler SG, Holmes CS, Goldstein DS et al. Neonatal diagnosis and treatment of Menkes disease. N Engl J Med Feb 7, 2008;358:605-614). (Reprints: Dr Kaler, National Institute of Child Health and Human Development, National Institutes of Health, Bldg 10, Rm 5-2571, 10 Center Dr, MSC 1832, Bethesda, MD 20892).

COMMENT. Menkes disease is an X-linked recessive infantile neurodegenerative disease caused by deficiency of a copper-transporting ATPase, ATP7A. Enzymes that require copper as a cofactor (dopamine-B-hydroxylase, cytochrome coxidase) are decreased. Symptoms are delayed for 6 to 8 weeks after birth. The disease is characterized by hypotonia, seizures, failure to thrive, and death by 3 years of age. Biochemical markers such as low serum copper and ceruloplasmin are unreliable in the neonatal period since they are low in normal neonates and overlap with the values found in Menkes disease. A molecular diagnosis, involving measurement of dopamine, norepinephrine and other catecholamines in plasma, is necessary to identify cases before symptoms develop and for copper replacement therapy to be successful. 